\title{
EMBEDDINGS BETWEEN DISCRETE WEIGHTED LEBESGUE SPACES WITH VARIABLE EXPONENTS
}

\author{
ALEŠ NEKVINDA
}

Abstract. Given mappings $p, q, v, w: \mathbb{Z} \rightarrow(0, \infty)$ we can consider discrete weighted Lebesgue spaces $\ell\left\{p_{n}\right\}\left(v_{n}\right)$ and $\ell\left\{q_{n}\right\}\left(w_{n}\right)$ with variable exponents. The necessary and sufficient condition to the $p, q, v, w$ for the embedding $\ell^{\left\{p_{n}\right\}}\left(v_{n}\right) \hookrightarrow \ell^{\left\{q_{n}\right\}}\left(w_{n}\right)$ is given.

Mathematics subject classification (2000): 46E30, $26 \mathrm{D} 15$.

Key words and phrases: Lebesgue spaces, variable exponent, embedding.

\section{REFERENCES}

[1] D. Cruiz-Uribe,A. Fiorenza And C. J. Neugebauer, The maximal function on variable $L^{p}$ spaces, Ann. Acad. Sci. Fenn. Math., 28, (1) (2003), 223-238.

[2] L. DiEnING, Maximal function on generalised Lebesgue spaces $L^{p(\cdot)}$, Ann. Acad. Sci. Fenn. Math., 7 , (2) (2004), 245-254.

[3] L. DIENING, Maximal function on Orlicz-Musielak spaces and generalized Lebesgue spaces, preprint, 2003, (2003).

[4] D. E. Edmunds, J. Lang And A. NeKvinda, On $\ell^{p(x)}$ norms, Proc. Roy. Soc. Lond. A, 455, (1999), 219-225.

[5] D. E. EDMunds, A. NEKVINDA, Averaging operators on $l^{\left\{p_{n}\right\}}$ and $L^{p(x)}$, Math. Inequal. Appl., 5, (2) (2002), 235-246.

[6] D. E. Edmunds, J. RÁKosník, Density of smooth functions in $W^{k, p(x)}(\Omega)$, Proc. Roy. Soc. Lond. A, 437, (1993), 153-167.

[7] D. E. EdMunds, J. RÁKOSNÍK, Sobolev embeddings with variable exponent, Studia Math., 143, (2000), 267-293.

[8] P. HarJulehto, P. HästÖ AND M. Pere, Variable exponent Lebesgue spaces on metric spaces: The Hardy-Littlewood maximal operator, Real Anal. Exange, 30, (1) to appeare.

[9] O. KovÁČIK, J. RÁKOSNíK, On spaces $L^{p(x)}$ and $W^{k, p(x)}$, Czechoslovak Math. J., 41, (1996), $167-177$.

[10] A. LERNER, Some remarks on the Hardy-Littlewood maximal function on variable $L^{p}$ spaces, preprint, 2004.

[11] A. NEKVINDA, Equivalence of $\ell\left\{p_{n}\right\}$ norms and shift operators, Math. Inequal. Appl., 5, (4) (2002), 711-723.

[12] A. NeKVINDA, Hardy-Littlewood maximal operator on $L^{p(x)}\left(\mathbb{R}^{n}\right)$, Math. Inequal. Appl., 7, (2) (2004), 255-265.

[13] A. NeKvinda, A note on maximal operator on $\ell^{\left\{p_{n}\right\}}$ and $L^{p(x)}(\mathbb{R})$, submitted to J. Funct. Spaces Appl., (2004)

[14] M. RŮŽIČKA, Electrorheological fluids: Modeling and mathematical theory Lecture Notes in Mathematics. 1748. Berlin: Springer, 2000.

[15] M. RŮŽIČKA, Flow of shear dependent electrorheological fluids, C.R.Acad. Sci. Paris Série, I 329, (1999), 393-398.

[16] S. G. SAMKO, The density of $C_{0}^{\infty}\left(\mathbb{R}^{n}\right)$ in generalized Sobolev spaces $W^{m, p(x)}\left(\mathbb{R}^{n}\right)$, Soviet Math. Doklady, 60, (1999), 382-385. 